Bulletin of the SeiImological Society of America. Vol. 64, No. 5, pp. 1383-1388. October 1974

\title{
HIGH-FREQUENCY ATTENUATION BY A THIN HIGH-VELOCITY LAYER
}

\author{
By George R. Mellman and Donald V. Helmberger
}

\begin{abstract}
The Cagniard-de Hoop method is used to investigate elastic-wave tunneling through a thin high-velocity layer. The results indicate high-frequency attenuation in the geometric shadow zone. Attenuation is roughly proportional to layer thickness. Comparison of results obtained by omitting the high-velocity layer but using an average $Q$ operator shows that effects of the high-velocity layer are similar to $Q$ effects, with the equivalent $Q$ for a given layer thickness varying as a function of source-receiver distance. These attenuation effects are explained in terms of deformation of the de Hoop contour.
\end{abstract}

\section{INTRODUCTION}

Classical ray theoretic techniques are often inadequate for the study of tunneling or diffraction effects. That is, under the assumptions of asymptotic ray theory no energy should be received in a shadow zone. Actual seismic data for the shadow zones resulting from the negative velocity gradients of the upper mantle low-velocity zone and the coremantle boundary show that long-period direct waves persist well into the shadow region, as is well known. This result indicates that diffraction effects are present and that a useful theory must properly take into account the curvature of wave fronts and realistic earth models.

In this study, we chose to investigate the problem of high-frequency attenuation caused by a thin high-velocity layer over a low-velocity zone, with a view to explaining the $12^{\circ}$ shadow zone. The model setup is given in Figure 1. We assumed a step-function point-source displacement potential and a flat Earth. Various thin layer thicknesses $h=0,0.5,5,10$ and $25 \mathrm{~km}$ were considered. This is perhaps the simplest geophysically meaningful diffraction problem. It was chosen largely to give insight into the workings of the Cagniard method in diffraction problems preparatory to exploring other more difficult, and geophysically more interesting, problems.

\section{SYNTHETICS}

The response for this two-layer system can be easily constructed by summing generalized rays (for example, see Helmberger, 1968). However, since $\mathrm{Th}_{3}$ is much larger than $\mathrm{Th}_{2}$, these rays can be grouped according to the number of times the bottom layer is traversed; that is, the effective reflection from the thin layer is constructed by summing the generalized rays reflected off the top and bottom boundaries of the thin layer and the many internally reflected multiples as displayed in Figure $2 \mathrm{a}$. The response returning from the bottom interface can be similarly constructed as displayed in Figure $2 b$. The next group of rays would contain the first multiple in the bottom layer. We are primarily concerned with the responses produced from the groupings given in Figure 2, especially $2 b$.

The first arrivals obtained at any of the distances in question are reflections off the thin layer. Intuitively, we feel that a thin layer should have little effect on a sufficiently long-period wave. The question arises, however, of what is meant by "sufficiently longperiod". Inasmuch as the reflection coefficient off the top of the layer is positive and the 
reflection coefficients for reflections off the bottom of the layer and for all upgoing multiple reflections are negative, we expect that the reflections will cancel for periods which are long compared to $2 h /(v \cos \theta)$ where $h=$ layer thickness, $v=$ velocity in thin layer, and $\theta$ is the angle from the vertical made by the incident wave. Unfortunately, this is valid only for angles much less than the critical angle. For responses from the top layer at distances where $\theta$ is well past the critical angle, we have absolutely no handle from ray theory on what sufficiently large may mean.

\begin{tabular}{|c|c|c|c|}
\hline \multicolumn{2}{|l|}{$\begin{array}{l}\text { POINT SOURCE } \\
*\end{array}$} & & $\begin{array}{r}\text { RECEIVER } \\
0\end{array}$ \\
\hline$T h_{1}=80 \mathrm{~km}$ & $\alpha_{1}=7$ & $\mathrm{~m} / \mathrm{sec}$ & $p_{1}=3.2$ \\
\hline$T h_{2}=h$ & $a_{2}=8$ & " & $\rho_{2}=3.4$ \\
\hline $\mathrm{Th}_{3}=80$ & $\alpha_{3}=7$ & $"$ & $p_{3}=3.2$ \\
\hline $\mathrm{Th}_{4}=0$ & $\alpha_{4}=8$ & " & \\
\hline
\end{tabular}

Fig. 1. Diagram illustrating the layered model. Model consists of two fluid layers sandwiched between two fluid half-spaces with source and receiver located $80 \mathrm{~km}$ above upper half-space boundary.
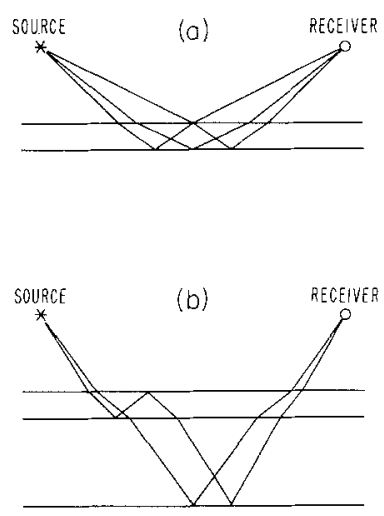

FIG. 2. (a) Diagram of generalized ray types considered in computing response for thin layer. In general, large numbers of multiple reflections must be considered in order to obtain convergence. (b) Generalized ray types considered in computing response from lower half-space. Only single reflections from half-space are considered because higher multiples are generally small and well removed in time from first reflections.

Reflections off the thin layer, as given by Figure 2a, were computed for $d=800$ and $h=0.5$ and 5. The results are shown in Figure 3. For $h=0.5$, the result is a highfrequency spike with width at half-height $(\mathrm{WHH})=0.1 \mathrm{sec}$. Figure 3 also indicates that using the Cagniard method on thin layers sometimes gives convergence problems, necessitating the use of prodigious numbers of generalized rays and large amounts of computer time. For $h=5$, the peak has broadened and doubled in height. This may be attributed to the presence, as expected, of a greater amount of the long-period components. WHH has increased to $0.25 \mathrm{sec}$. It should be noted that Figure 3 contains refractions as well as reflections, and that the small precursor in Figure $3 \mathrm{~b}$ is probably the result of refraction of long-period components, not numerical noise. Some of these features have been studied previously (see Dunkin, 1963).

Synthetics based on the rays given by Figure $3 b$ are displayed in Figure 4 for various values of $h$. For models with thin layer thickness $h=0$, the critical angle for reflections from the half-space is reached at $d=575 \mathrm{~km}$. Thus, first arrivals for distances $d=800$ and $1,200 \mathrm{~km}$ will be refractions. Simple ray theoretic calculations show that, for a highvelocity layer of arbitrarily small thickness, these refractions will be suppressed. This is 
clearly a nonphysical result, because long-period waves should not be affected by a layer of finite velocity with a thickness that is small compared to this wavelength.

Synthetic seismograms generated using Cagniard calculations (Figure 4) indicate that sufficiently long periods are, in fact, insensitive to the presence of the thin high-velocity layer. For $h=0$, we have the usual result for a layer over a half-space. For $d=400 \mathrm{~km}$,

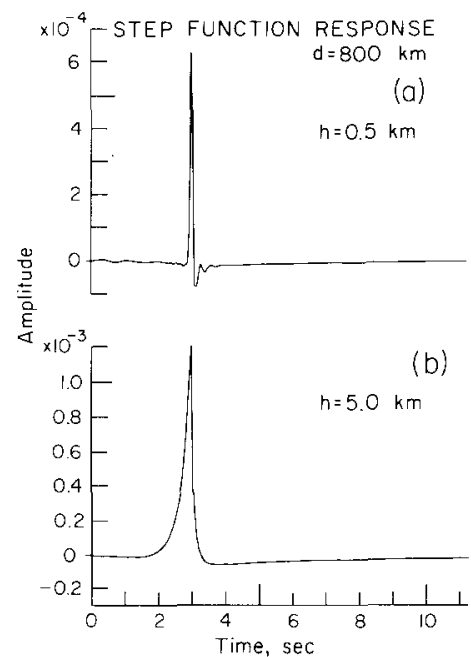

FIG. 3. Displacement potential response for thin layer at $d=800 \mathrm{~km}$. In each case, 15 generalized rays were summed up to obtain synthetic seismogram. Note increase in amplitude and width of response as $h$ is increased. In all cases, arrivals from thin layer occur well before arrivals from half-space.
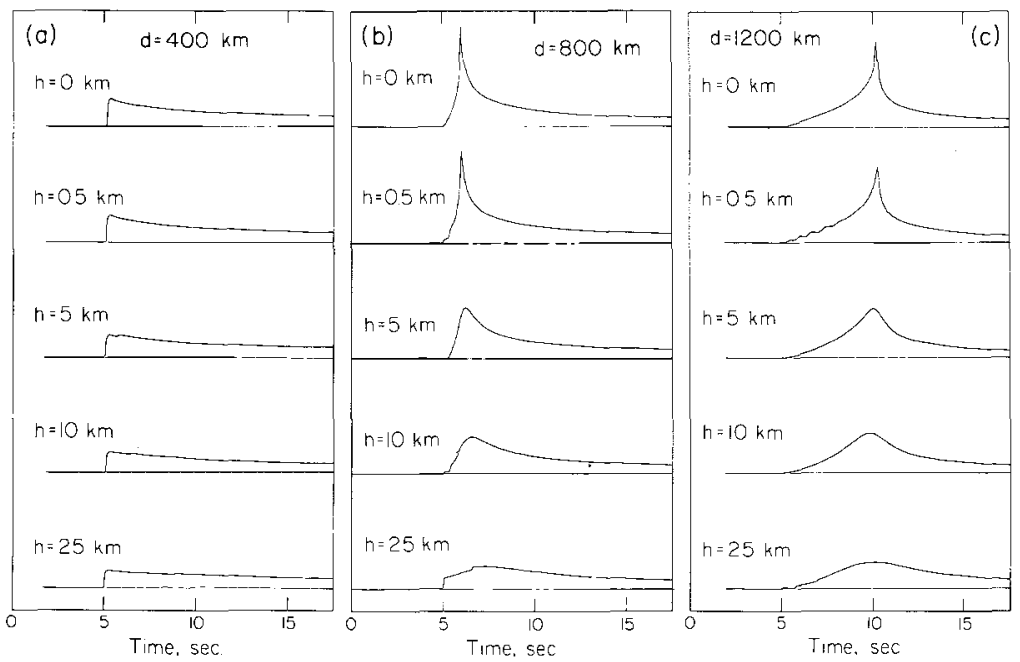

Fig. 4. Displacement potential response for rays reflected from lower half-space. In all cases, reduced travel times have been used. All amplitudes have been scaled to amplitude of $3=800, h=0$ synthetic.

the wave shape is essentially unaltered from the shape of the source-time function. As $d$ increases, the reflection angle increases past the critical angle and a refraction becomes apparent. By $d=1,200 \mathrm{~km}$, the refraction is quite well developed. Addition of the thin, high-velocity layer has the effect of attenuating high frequencies, with progressively greater attenuation as $h$ is increased. It should also be noted that for a fixed value of $h$, attenuation increases as $d$ increases. 
The behavior of this attenuation effect as a function of distance $d$ suggests that, observationally, effects which are due to a thin high-velocity layer could be confused with more normal attenuation effects. To investigate this possibility, average $Q$ operators (Carpenter, 1967) for a variety of $Q$ values were applied to synthetic seismograms generated for the case $h=0$ at $d=400,800$ and $1,200 \mathrm{~km}$. The resultant seismograms were then compared to the synthetics obtained for nonzero values of $h$. In this way, it was possible to associate an equivalent $Q$ value with each $h$ at a given distance $d$ (Table 1 ). In general, for a given value of $h$, the equivalent $Q$ values decrease as distance is increased. Thus, for the distance range studied, attenuation increases slightly faster for effects which are due to the thin layer than for the average $Q$ operator.

TABLE 1

EquiValent $Q$ VAlues

\begin{tabular}{crrr}
\hline $\begin{array}{c}\text { Thin Layer } \\
\text { thickness } \\
h(\mathrm{~km})\end{array}$ & \multicolumn{3}{c}{ Source-Receiver Distance $d(\mathrm{~km})$} \\
\cline { 2 - 4 } & 400 & 800 & 1200 \\
\hline 0.5 & $>2500$ & 2000 & 2000 \\
5 & 500 & 400 & 300 \\
10 & 250 & 175 & 150 \\
25 & $<100$ & $<100$ & $<100$ \\
\hline
\end{tabular}

A better understanding of the attenuation effects of the thin, high-velocity layer may be obtained by examining the de Hoop contours for this problem.

Assuming a step-function source, an approximate response for a primary ray, reflecting off the bottom interface, can be written

$$
\phi(r, z, t)=\sqrt{\frac{2}{r} \frac{1}{\pi}}\left[\frac{1}{\sqrt{ } t} *\left(\operatorname{Im} \frac{\sqrt{p}}{\eta_{1}} R(p) \frac{d p}{d t}\right)\right]
$$

where $R(p)$ is the product of all transmission and reflection coefficients (see Helmberger, 1968). The above function is to be evaluated along the contour described by

$$
t=p r+2 \sum_{j=1}^{3} T h_{j} \eta_{j} ; \eta_{j}=\left(\frac{1}{\alpha_{j}^{2}}-p^{2}\right)^{1 / 2} .
$$

Example contours for rays that are transmitted directly through the thin layer are displayed in Figure 5 . The function $(d p / d t)$ can be approximated by assuming the contours to be vertical straight lines with

$$
\frac{d p}{d t} \approx i\left(t-t_{R}\right)^{-1 / 2}\left(2 \frac{d^{2} t}{d p^{2}}\right)^{-1 / 2}
$$

where

$$
\frac{d^{2} t}{d p^{2}}=2 p \sum_{j=1}^{3} \frac{T h_{j}}{\eta_{j}^{3} d_{j}^{2}}
$$

and $t_{R}$ is the reflection time. The above approximation leads to

$$
\frac{1}{\sqrt{ } t} * \frac{1}{\sqrt{t-t_{R}}}=\pi H\left(t-t_{R}\right)
$$

which corresponds to geometric rays and is equivalent to the well-known saddle-point approximation. 
The fact that, even in the $h=0$ case where no tunneling effects occur, the de Hoop contours differ from straight lines implies that, even in the simple case of a layer over a half-space, frequency-dependent effects are present.

In the case $d=400$ (Figure 5), the principal effect of the addition of the high-velocity layer is to shift the de Hoop contour to the left. This is caused by changes in the geometric ray parameter due to changes in the geometry of the problem. Because the shape of the contour is unchanged once it has left the real axis, we expect that $d p / d t$ will be little changed as $h$ is increased, and that the shape of the time domain response also will be little changed. Such changes as do occur are caused by changes in the generalized reflection coefficient due to changes in the geometric ray parameter.

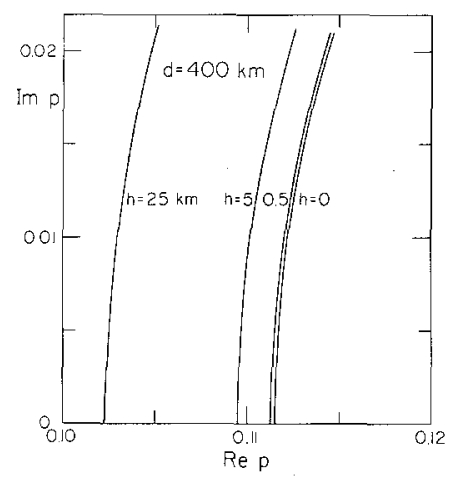

FIG. 5. de Hoop contours for $d=400 \mathrm{~km}$. The units of $p$ are sec $/ \mathrm{km}$.

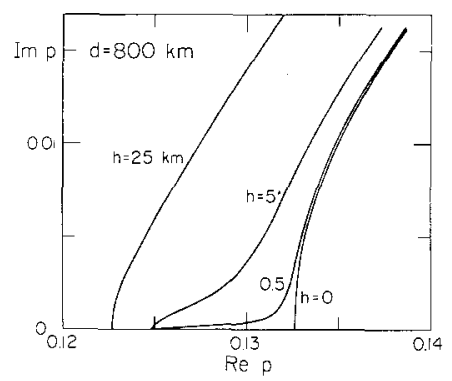

Fig. 6. de Hoop contours for $d=800 \mathrm{~km}$.

As the critical angle is reached, however, contour changes assume greater importance. This may be seen by examining Figure 6 . For $h=0$, the contour follows the real axis past the branch point at $p=1 / 8$. At this point, the reflection coefficient becomes imaginary and the refraction begins. The contour continues along the real axis until the saddle point is reached, where it breaks vertically. This corresponds to the end of the refraction and the beginning of the reflection. The contour then begins to bend over toward the real axis, as it did in the $d=400$ case.

When the high-velocity layer is added, the presence of the term $\eta_{2}=2 h\left(1 / 8^{2}-p^{2}\right)^{1 / 2}$ in $t$ moves the saddle point to the left of $p=1 / 8$. This forces the contour to leave the real axis before the branch at $p=1 / 8$, and hence the refraction is suppressed. For small values of $h$, even though the contour still leaves the real axis at right angles, it quickly bends over to nearly parallel the axis. This gives rise to a pseudo-refraction and our tunneling effect. As the contour approaches the saddle point for the $h=0$ case, it bends upward. This corresponds to an increase in $d p / d t$, and hence the arrival of the reflection 
in the $h=0$ case. The change in the shape of the contour becomes more gradual as $h$ is increased and the pseudo-refraction portion of the contour is moved farther from the real axis. This implies that as $h$ is increased, $d p / d t$ becomes a more slowly varying function of it. It is the fact that gives rise to the attenuation effect observed in Figure 3. As $t$ further increases, the contours begin to parallel the $h=0$ contour. This implies that long-period energy is little effected by addition of the thin layer. As $d$ is further increased (Figure 7), the above effects remain qualitatively the same, but become more pronounced because of increases in the length of the pseudo refraction.

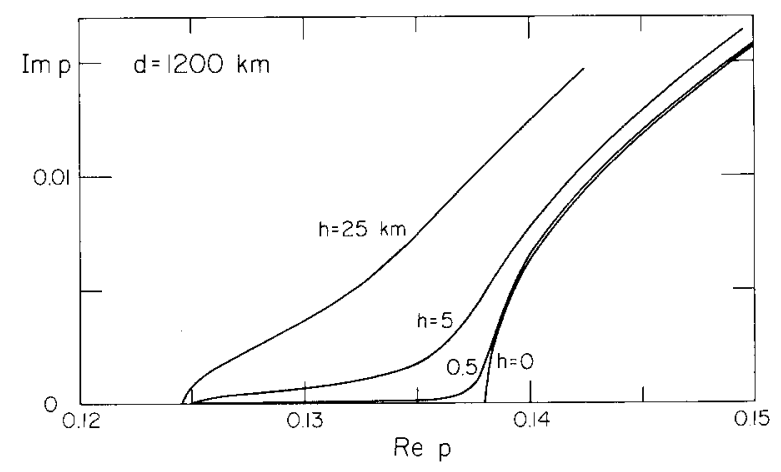

FIG. 7. de Hoop contours for $d=1200 \mathrm{~km}$.

\section{CONCLUSIONS}

Numerical experiments on nongeometrical ray responses were conducted to test the feasibility of treating tunneling problems by the application of the Cagniard-de Hoop method. The results indicate that the technique is well suited to handle those problems if one takes certain precautions; namely, detailed shapes of contours must be determined as well as the inclusion of multiples when rays are traveling nearly horizontally.

Tunneling through a thin high-velocity layer was found to be nearly equivalent to an application of a $Q$ operator. The de Hoop contours are displaced off the real axis in much the same fashion as allowing complex velocity. The effect is to remove the high frequencies as the layer thickness is increased. This allows the characterization of effective $Q$ in terms of layer thickness although there is a small angular dependence as expected.

\section{ACKNOWLEDGMENTS}

This research was supported by the Advanced Research Projects Agency of the Department of Defense and was monitored by the Air Force Office of Scientific Research under Contracts F44620-72-C-0078 and F44620-72-C-0083.

\section{REFERENCES}

Carpenter, E. W. (1967). Teleseismic signals calculated for underground, underwater, and atmospheric explosions, Geophysics 32, 17-32.

Dunkin, J. W. (1963). A study of two-dimensional head waves in fluids and solid systems, Geophysics 28, $563-581$.

Helmberger, D. V. (1968). The crust-mantle transition in the Bering Sea, Bull. Seism. Soc. Am. 58, 179214.

Seismological Laboratory

Division of Geological and Planetary Sciences

California Institute of Technology

Pasadena, California 91109.

Contribution No. 2455.

Manuscript received March 21, 1974. 